\title{
Impact of Sleeve Gastrectomy on Subjective Hunger and Appetite Ratings and Endocrine Hormones
}

\author{
Avelino De Leon \\ University of Chicago Department of Medicine \\ Jeremy White \\ University of Chicago Department of Medicine \\ Isabel Casimiro \\ University of Chicago Department of Medicine \\ Amarachi Okoli \\ University of Chicago Department of Medicine \\ Matthew J. Piron \\ University of Chicago Department of Medicine \\ Matthew J. Brady \\ University of Chicago Department of Medicine \\ Erin Hanlon ( $\sim$ ehanlon@medicine.bsd.uchicago.edu ) \\ University of Chicago Department of Medicine https://orcid.org/0000-0002-1914-9724
}

\section{Research Article}

Keywords: Bariatric Surgery, Weight-loss, Food Choice, Subjective, Hunger, Appetite, Ratings, Food Intake, Endocrine Hormones

Posted Date: January 25th, 2022

DOI: https://doi.org/10.21203/rs.3.rs-1182069/v1

License: (c) (i) This work is licensed under a Creative Commons Attribution 4.0 International License.

Read Full License 


\section{Abstract}

Background: The effects of vertical sleeve gastrectomy (VSG) surgery on the levels of patients' hunger and appetite across the day and relation to weight loss achieved were examined.

Methods: Visual analog scale (VAS) hunger and appetite ratings were digitally collected from 10 female participants across a day during in-lab sessions 1-2 weeks before and 12 weeks after surgery. Fasting hormone levels were also assessed in 8 participants.

Results: Post-surgery ratings were reduced overall despite fasting levels of leptin and ghrelin changing in favor of increased food intake. Notably, the majority of ratings from before breakfast were not different from ratings after dinner, suggesting that VSG induces changes that overcome the homeostatic drive to oppose a hypocaloric diet.

Conclusions: These findings highlight the effectiveness of sleeve gastrectomy surgery in curbing foodseeking behavior despite a negative calorie balance.

\section{Introduction}

Vertical sleeve gastrectomy (VSG) surgery is extremely effective in reducing body weight and associated co-morbidities [1, 2]. A major axis perturbed following VSG is the regulation of energy homeostasis by the endocrine hormones: leptin and ghrelin. Leptin is secreted predominantly by adipose tissue in proportion to the amount of lipids stored and its effects on satiety are mediated indirectly through the central nervous system. In contrast, ghrelin is secreted in pulses across the day from specialized cells in the stomach and the upper small intestine [3, 4]. Progressive weight-loss through caloric restriction has been observed to lead to a decline in the levels of serum leptin as a result of decreased adipose tissue mass [5]. Conversely, VSG has been shown to lower serum ghrelin levels after surgery due to the postulated removal of a majority of ghrelin producing cells $[6,7]$. The simultaneous decline of both hormones in the axis leads to an unclear understanding of how or even if hunger and appetite are affected by VSG surgery[8].

Hunger is thought to be driven more by homeostatic endocrine signaling mechanisms, while appetite is understood to be motivated more by hedonic reward pathways. Bariatric surgery may alter the hedonic or rewarding properties of food and that may contribute to changes in hunger, appetite, food preference, and actual consumption [9-11]. The decline in food intake following VSG may also result from the combination of food capacity restriction and alterations in circulating endocrine hormones in opposition to food intake $[12,13]$.

The purpose of the current study was to examine the relationship between active weight-loss, subjective ratings of hunger and appetite across a day, and fasting levels of the hormones leptin and ghrelin in women undergoing VSG surgery. We hypothesized that, at twelve weeks post-surgery before sustained weight loss has been achieved, subjective ratings of hunger and appetite would be reduced overall 
compared to pre-surgical baseline ratings. In addition, we hypothesized that these ratings would be impacted less by ghrelin activity and more by caloric intake.

\section{Materials And Methods}

\subsection{Subject Characteristics}

Screening of potential subjects and the process of enrollment into the clinical study was conducted as previously described [14]. VSG was the most common bariatric surgical procedure performed at the University of Chicago thus we only enrolled those underdoing this type of bariatric surgery. Further, women accounted for $80 \%$ of patients undergoing the procedure therefore we have only included women in the current study in order to achieve sufficient power for analysis. Fourteen women with class II obesity with co-morbidities or class III, IV, or V obesity were voluntarily enrolled following approval and scheduling for laparoscopic VSG surgery. Those with class II obesity with co-morbidities did not have metabolic comorbidities detailed in our exclusion criteria. Subjects were screened for the absence of malignant physiological or endocrine conditions, pregnancy, irregular lifestyles, and uncontrolled obstructive sleep apnea. De-identified subject characteristics are listed (Table 1).

Table 1

De-identified Subject Characteristics

\begin{tabular}{|llccc|}
\hline Subjects & $\mathbf{N}=\mathbf{1 0}$ & Mean Age (years) & & $\mathbf{3 7 \pm 1 0 . 1}$ \\
\hline Race & 8 AA, 2 NHW & Mean Height $(\mathrm{cm})$ & & $168.4 \pm 4.6$ \\
\hline Biometric & Timepoint & Mean & SEM & p-value \\
\hline Weight $(\mathrm{kg})$ & Baseline & 129.9 & \pm 5.1 & \\
\hline BMI $\left(\mathrm{kg} / \mathrm{m}^{2}\right)$ & Post-surgery & 109.5 & \pm 5.3 & $1.2 \mathrm{E}-09$ \\
\hline & Baseline & 45.6 & \pm 1.8 & \\
\hline Waist:Hip & Post-surgery & 38.3 & \pm 1.9 & $1.2 \mathrm{E}-08$ \\
\hline Body Fat Mass (\%) & Baseline & 0.924 & \pm 0.021 & \\
\hline & Post-surgery & 0.886 & \pm 0.030 & 0.28 \\
\hline & Baseline & 53.5 & $\pm 0.8 \%$ & \\
\hline
\end{tabular}

AA: African-American; NHW: Non-Hispanic White. Height and age were measured at intake screening appointment. Weight was measured with subject vitals at each intake appointment. Subject BMI, waist:hip ratio, and percent body fat mass were recorded the mornings of baseline and post-surgery laboratory sessions. Statistical significance calculated by two-tailed paired T-Test.

\subsection{Laboratory Sessions}


Subjects were admitted 1-2 weeks prior to the scheduled surgery date (baseline) and 12-13 weeks following surgery (post-surgery), when there are no longer post-surgery food restrictions and during active weight loss, rather than after a stable new weight has been achieved. Bioimpedance was assessed using the Quantum X BIA analyzer validated in this population as previously described [15]. Body mass index (BMI), percent body fat mass, and waist:hip ratios were recorded during the morning of baseline and postsurgery in-laboratory sessions. In-lab sessions were structured as previously reported [14]. During in-lab sessions, four isocaloric mini-meals were provided per day. Total calories offered to each subject during baseline sessions was determined according to the Mifflin-St. Jeor equation [16]. At post-surgery lab sessions, all subjects were offered $1000 \mathrm{kcal}$ per day distributed over four mini-meals of $250 \mathrm{kcal}$ each, based on post-surgical guidelines. A registered dietitian from the Clinical Resource Center Metabolic Kitchen supervised the preparation of all meals and subjects were given thirty minutes to eat. If the entire meal was not consumed, leftover food was weighed to determine calories and macronutrients consumed (Table 2). For both sessions, mini-meals were served at 0900, 1230, 1530, and 1900 hours. One subject was provided an incorrect meal for their first post-surgery in-lab dinner (450kcal, 200 more kcal than intended). Data for this subject and this meal were not excluded since its removal did not significantly change findings.

Table 2

Laboratory Session Diet \& Food Consumption Information

\begin{tabular}{|c|c|c|c|c|}
\hline Macronutrient & Timepoint & Mean & SEM & p-value \\
\hline \multirow[t]{2}{*}{ Total Food Eaten (g) } & Baseline & 1920 & \pm 109 & \multirow[t]{2}{*}{ 9.7E-08 } \\
\hline & Post-surgery* & 583 & \pm 76 & \\
\hline \multirow[t]{2}{*}{ Calories (kcal) } & Baseline & 2223 & \pm 168 & \multirow[t]{2}{*}{ 2.7E-06 } \\
\hline & Post-surgery* & 591 & \pm 90 & \\
\hline \multirow[t]{2}{*}{ Protein (g) } & Baseline & 103.8 & \pm 4.9 & \multirow[t]{2}{*}{$9.5 \mathrm{E}-07$} \\
\hline & Post-surgery* & 48.0 & \pm 4.6 & \\
\hline \multirow[t]{2}{*}{ Carbohydrate (g) } & Baseline & 278.9 & \pm 22.4 & \multirow[t]{2}{*}{$1.7 \mathrm{E}-06$} \\
\hline & Post-surgery* & 60.7 & \pm 9.5 & \\
\hline \multirow[t]{2}{*}{ Fat (g) } & Baseline & 79.5 & \pm 7.3 & \multirow[t]{2}{*}{ 2.3E-05 } \\
\hline & Post-surgery* & 17.5 & \pm 4.6 & \\
\hline
\end{tabular}

Four isocaloric meals were prepared for each subject for each day of laboratory sessions. Proportion of macronutrients matched between otherwise identical laboratory sessions at baseline or post-surgery. Total calories offered at baseline were calculated according to the Mifflin-St. Jeor equation. Subjects were given thirty minutes to eat. Leftover food was weighed to calculate calories consumed. Statistical significance calculated by two-tailed paired T-Test. * One subject received one incorrect meal the night before the full day of surveys; this data is included in the analysis. 


\subsection{Fasting Serum and Plasma Hormone Levels}

Morning fasting blood samples were collected. Acylated ghrelin and total ghrelin were measured via ELISA (Millipore Sigma EZGRT-89K, EZGRA-88K) with a lower limit of detection $8 \mathrm{pg} / \mathrm{mL}$ and CV of 3.5$6.6 \%$. Leptin was measured via radioimmunoassay (Millipore Sigma HL-81K) with a lower limit of detection $0.5 \mathrm{ng} / \mathrm{mL}$ and $\mathrm{CV}$ of $3.6-6.2 \%$.

\subsection{Assessment of Hunger \& Appetite}

During laboratory sessions, subjects completed four validated, computerized visual analog scales (VAS) to assess hunger and appetite at eight time points: 25 minutes before, and 1hour and 35 minutes after each of the four mini-meals. With computerized VAS $(0 \mathrm{~cm}$, Not at all, to $10 \mathrm{~cm}$, Extremely or A very large amount), subjects reported their levels of hunger, at the time of the questionnaire, described [17].

To assess appetite at each time point, subjects used computerized VAS to rate how much they would enjoy eating foods, at the time of the questionnaire, from 7 different categories [17]. Composite appetite results were computed arithmetically by summation of the respective basic appetite categories.

Composite appetite categories were: A) Global Appetite: all 7 categories; B) High Carb-High-Fat (HCHF); C) High Carb-Low-Fat (HCLF); D) Sweet \& Starchy; and E) High Protein.

\subsection{Mixed Model Construction}

Mixed modeling was utilized to estimate the degree of difference in ratings between baseline and postsurgery. Model fitting was conducted by R package (Ime4). In order to ensure that the dependence derived from time series collection was accounted for in the statistics, the random vector was a simple intercept set as the Subject Identification Number. Experimental ratings were unaltered at entry into the data set.

The surgery-associated parsimonious model was calculated by using surgery as a binomial variable (Pre [0], Post [1]) and iterative inclusion of factors representing potential corrections for: 1) age (integer, years), 2) pre-surgery weight loss, 3) post-surgery weight loss, or 4) time of day. Given the known effect of meals to affect hunger and appetite, we represented time of day as the best combination of factors including 1 ) prandial (binomial, pre-meal [0], post-meal [1]); and 2) time point (integer, $\{1,2, \ldots, 8\}$ ), meal (integer, $\{1,2,3,4\}$ ), latter part of day (binomial, first half [0], latter half [1]). To allow for correction by weight loss, it was held constant for all respective points of a given subject. An enhanced model was chosen by: 1) an improved Akaike Information Criterion of the $\mathrm{N}+1$ model (compared to $\mathrm{N}$ model without the addition of the factor), 2) each factor effect has low Type $S$ error rate $(p<0.05)$, and low inflation of standard error of estimation $(<50 \%)$.

\section{Results}

\subsection{Laboratory Session Calorie \& Macronutrient Intake}


Ten of the fourteen women enrolled in the study completed the required minimum of 5 out of 8 surveys during both baseline and post-surgery lab sessions. The remaining 4 subjects did not complete sufficient number of surveys in either baseline and/or post-surgery to be included in the model for analysis.

Post-surgery, subjects consumed on average significantly fewer calories per day than at baseline $(\mathrm{p}=$ 2.7e-06), and also fewer than the $1000 \mathrm{kcal}$ offered. Average baseline quantities of protein, carbohydrates, and fat consumed represent, respectively, $18.7 \%, 50.1 \%$, and $32.2 \%$ of average total calories at baseline. Post-surgery average quantities of protein, carbohydrate, and fat consumed represent, respectively, $32.3 \%, 41.1 \%$, and $26.6 \%$ of average total calories at post-surgery. Total macronutrient intake was significantly decreased post-surgery $(p=9.5 e-07,1.7 e-06$, and $2.3 e-05$ for protein, carbohydrate, and fat, respectively; Table 2). For the following data analyses, linear mixed modeling was used throughout.

\subsection{Subjective Ratings of Hunger \& Appetite 3.2.1. Surgery Effect}

Surgery was revealed to significantly decrease ratings of appetite for high-carbohydrate, low-fat (HCLF) foods (HCLF: $p=0.018)$ and of global appetite $(p=0.038)$, Figure 1A. Specifically, appetite ratings were significantly decreased for protein $(p=0.047)$, fruits $(p=2.7 e-4)$, and vegetables $(p=0.023)$, Figure $1 B$. With regard to subjective ratings of hunger, surgery was revealed to significantly decrease ratings of thirst $(p=5.4 e-3)$ and quantity can eat $(p=1.6 e-3)$, and significantly increased ratings of nausea $(p=0.046)$, Figure $1 \mathrm{C}$.

\subsubsection{Weight-loss}

Greater weight-loss was predicted by significant decreases in average ratings of global appetite $(p=1.1 e-$ 4), appetite for high-carbohydrate, high-fat (HCHF) foods $(p=1.9 e-4), \operatorname{HCLF}$ foods $(p=1.9 \mathrm{e}-4)$, fruits \& sweets $(p=2.2 e-4)$, and high-carbohydrate foods $(p=6.8 \mathrm{e}-6)$, Figure 1D. Specifically, greater weight-loss was predicted by changes in average ratings of appetite for fruits $(p=2.2 e-30.01)$ and for sweets $(p=$ $0.043)$, Figure 1E. With regard to subjective ratings of hunger, greater weight-loss was predicted by changes in both quantity can eat $(p=6.7 e-4)$ and nausea $(p=0.05)$, Figure $1 F$.

\subsubsection{Calories Eaten}

A significant decrease in average ratings of appetite for HCLF foods was predicted by the decrease in calories eaten $(p=0.028)$, Figure 2A, specifically, in ratings fruits $(p=5.0 e-3)$, Figure 2B. The decrease in calories eaten predicted significant decreases in ratings of quantity can eat $(p=7.6 e-4)$ and increases in ratings of nausea $(p=0.034)$, Figure $2 \mathrm{C}$.

\subsubsection{Survey Responses Across Time}

The influence of time across the day was a predictor of changes in ratings of appetite for high protein (HP) foods ( $p=2.9 e-4)$, sweet \& starchy (SS) foods ( $p=0.046)$, HCLF foods ( $p=7.9 e-4)$, and of global appetite $(p=6.5 \mathrm{e}-4)$, Figure 3A. Specifically, later-in-the-day surveys could predict decreases in ratings of 
appetite for dairy $(p=0.017)$, protein $(p=5.5 e-6)$, fruits $(p=0.023)$, starchy $(p=5.2 e-3)$, salty $(p=0.045)$, and vegetables $(p=9.4 \mathrm{e}-4)$, Figure 3B. In parallel, time was revealed to predict significant decreases in ratings of hunger $(p=1.1 \mathrm{e}-4)$, thirst $(p=0.020)$, desire to eat $(p=7.4 \mathrm{e}-4)$, and quantity can eat $(p=1.2 \mathrm{e}-3)$. Time across the day was also shown to predict a significant increase in ratings of Satiety $(p=1.2 e-6)$, and no significant change in ratings of nausea (Figure 3C).

\subsubsection{Survey Responses at Baseline \& Post-Surgery}

Significant changes in the difference between average ratings at baseline and post-surgery were observed for the hunger categories of hunger $(p=8.6 e-3)$, thirst $(p=0.013)$, satiety $(p=0.041)$, and quantity can eat $(p=7.0$ e-5), Figure 4A. Specifically, the significant differences between ratings were observed early in the day (i.e. before the first meal; Fasting), with no significant differences observed between ratings after the last meal (Last; Figures 4B-4G).

Significant decreases in ratings of appetite at baseline and post-surgery were observed for the categories of: global appetite $(p=1.3 e-3), \operatorname{HCLF}(p=1.6 e-4), \operatorname{HP}(p=8.1 e-5)$, Figure $5 A$. The specific food types for which appetite ratings were significant decreased were starchy $(p=2.4 \mathrm{e}-3)$, dairy $(p=0.034)$, protein $(p=$ $2.5 e-4)$, and vegetables $(p=1.3 e-3)$, Figure 5B. As with hunger survey responses, significant differences between ratings at baseline and post-surgery were observed early in the day, with the addition that ratings of appetite for fruits were also significantly different after the last meal (Figures 5C-5N).

\subsection{Hormone Levels}

\subsubsection{Serum Leptin \& Ghrelin}

Serum leptin and ghrelin were measured in 8 of the subjects. The percent of fasting acylated ghrelin was significantly increased post-surgery relative to baseline $(p=0.014)$, Figure 6A. In parallel, fasting serum leptin was found to be significantly decreased post-surgery $(p=6.7 e-4)$, Figure 6B. Linear modeling revealed that post-surgery percent weight-loss was correlated with the changes in: fasting serum leptin ( $p$ $=1.5 \mathrm{e}-10)$, the fasting percent ratio of acylated to des-acylated ghrelin $(p=8.3 \mathrm{e}-3)$, and calories eaten $(p=$ $5.8 \mathrm{e}-4)$, Figure $6 \mathrm{C}$. The ratio of fasting leptin to total fasting ghrelin was significantly decreased postsurgery relative to baseline $(p=0.030$; data not shown).

\subsubsection{Hunger \& Appetite}

The relationships between serum leptin and ghrelin levels and subjective ratings of hunger and appetite were estimated. Specifically, given average hormone levels at baseline and post-surgery and the association derived from the trained model, the expected survey ratings were approximated. As predicted, there was a decline in circulating leptin levels 12 weeks after surgery. The only significant difference between average baseline and post-surgery ratings that was associated with the decrease in serum leptin was for Quantity Can Eat (Figure 6D-6F). Furthermore, no significant differences between average baseline and post-surgery ratings of hunger and appetite were predicted by the increase in the percent ratio of acylated ghrelin to des-acylated ghrelin (\%AG/DAG; Figure 6G-6I). 


\section{Discussion}

The current study measured changes in subjective ratings of hunger and appetite and endocrine appetite hormones in women with obesity undergoing VSG surgery. Post-surgery fasting serum levels of the hormones leptin and ghrelin were altered in favor of increased food intake, despite subjective ratings of hunger and appetite being reduced overall. In addition, a majority of baseline ratings of hunger and appetite were declined over the course of the laboratory session, but post- surgery most ratings of hunger and appetite were low in the morning and relatively constant over the day.

Greater weight loss was associated with decreases in particular ratings of hunger and appetite, especially for ratings of Global Appetite, HCHF, HCLF, Fruits, Sweets, and High Carb foods. A significant trend was also seen for hunger ratings of Quantity Can Eat and Nausea. The drop in Calories Eaten was significantly associated with decreases in ratings for HCLF, Fruits, and Quantity Can Eat, and with an increase in ratings of Nausea. Taken together, these results suggest that VSG surgery may have a greater impact on subjective appetite than on subjective hunger, in line with previous studies [9]. VSG surgery may mask the effects of homeostatic endocrine hormones potentially through changes in gut-brain communication, the sympathetic nervous system, and neuroendocrine signaling.

The effects of bariatric surgery on leptin and ghrelin have been reported by a number of studies [12, 1822]. In agreement with other reports [5, 23, 24], linear modeling revealed that percent weight lost correlates positively with the degree of change in fasting serum leptin, suggesting that these individuals are more leptin sensitive 12 weeks post-surgery. In contrast, while serum levels of acyl, des-acyl, and total ghrelin appeared to be higher post-surgery, only the percent-ratio \%AC/DAG was significantly increased, but did not correlate with changes in hunger and appetite ratings. These findings may suggest a compensatory response during active weight loss after VSG similarly to diet induced weight loss [25]. However, the impact of leptin and ghrelin on ratings and food intake may be masked or otherwise modulated by changes in the levels of other gastrointestinal and pancreatic peptide hormones not investigated in the current study. Our study had some limitations: the current study only evaluated fasting serum hormone levels. Future research should assess serum hormone levels in parallel with hunger and appetite over a 24-hour period. Ten participants completed assessments at baseline and 12 weeks post-surgery that allowed for sufficiently powered analysis, for which we observed significant findings. To that end, fasting hormone levels were assessed pre and post-surgery in only 8 of the participants. Even though we did observe significant differences in both leptin and ghrelin serum concentrations with this within-subject design, future experiments would benefit from obtaining a larger sample size.

In conclusion, the current findings support the notion that sleeve gastrectomy surgery leads to comparable levels of hunger and appetite as observed prior to surgery, even with the intake of fewer calories, and highlights the effectiveness of sleeve gastrectomy surgery in curbing food-seeking behavior despite a very steep negative calorie balance.

\section{Abbreviations}


BMI - Body Mass Index; VSG - Vertical Sleeve Gastrectomy; kcal - kilocalorie; ELISA - Enzyme-Linked Immunosorbent Assay; VAS - Visual Analog Scale; HCLF - High-Carbohydrate, Low Fat; HCHF - HighCarbohydrate, High-Fat; HP - High Protein; SS - Sweet \& Starchy; AG - acylated ghrelin; DAG - desacylated ghrelin; GLP-1 - glucagon-like-peptide-1

\section{Declarations}

Acknowledgments: We thank Dr. Eve Van Cauter, PhD for her guidance and expertise, the University of Chicago Medicine Clinical Research Center and Sleep Research Lab staff for their help in carrying out these intricate studies, the Research Assistants for their help and dedication to our project, and the study volunteers.

Funding: This research was funded by NIH grants, T32 HL007909 (JW), R01 DK103014 (MJB), and P30 DK028595 (MJB; University of Chicago Diabetes Research \& Training Center).

Conflicts of Interest: The authors declare no conflicts of interest and have no relevant financial or nonfinancial interests to disclose.

Author Contributions: Conceptualization, ECH and MJB; methodology, JW and ECH; validation, $A D, J W$, and $\mathrm{ECH}$; formal analysis, $\mathrm{AD}$ and JW; investigation, $\mathrm{AD}, \mathrm{JW}, \mathrm{IC}, \mathrm{MJP}$, and $\mathrm{ECH}$; resources, MJB and ECH; data curation, $A D, J W, I C, A O$, and MJP; writing-original draft preparation, $A D$ and JW; writing-review and editing, AD, JW, IC, AO, MJP, MJB, ECH; visualization, AD and JW; supervision, MJB and ECH; project administration, MJB and $\mathrm{ECH}$; funding acquisition, $\mathrm{MJB}$ and $\mathrm{ECH}$.

Data Availability: The datasets generated during and/or analyzed during the current study are available from the corresponding author on reasonable request.

Ethics and Consent: The studies were approved by the Institutional Review Board of The University of Chicago (IRB\#14-0984), and all participants were compensated for their participation. All participants provided written informed consent in accordance with the Declaration of Helsinki.

\section{References}

1. Arterburn DE, Telem DA, Kushner RF, Courcoulas AP (2020) Benefits and Risks of Bariatric Surgery in Adults: A Review. JAMA 324(9):879-887. doi:10.1001/jama.2020.12567

2. Wolfe BM, Kvach E, Eckel RH (2016) Treatment of Obesity: Weight Loss and Bariatric Surgery. Circ Res 118(11):1844-1855. doi:10.1161/circresaha.116.307591

3. Makris MC, Alexandrou A, Papatsoutsos EG, Malietzis G, Tsilimigras DI, Guerron AD et al (2017) Ghrelin and Obesity: Identifying Gaps and Dispelling Myths. A Reappraisal. In Vivo, 31(6), 1047-1050. doi:10.21873/invivo.11168

4. Nunez-Salces M, Li H, Feinle-Bisset C, Young RL, Page AJ (2020) The regulation of gastric ghrelin secretion. Acta Physiol (Oxf) e13588. doi:10.1111/apha.13588 
5. Friedman JM (2019) Leptin and the endocrine control of energy balance. Nat Metab 1(8):754-764. doi:10.1038/s42255-019-0095-y

6. Steinert RE, Peterli R, Keller S, Meyer-Gerspach AC, Drewe J, Peters T et al (2013) Bile acids and gut peptide secretion after bariatric surgery: A 1-year prospective randomized pilot trial (Article). Obesity 21(12):E660-E668. doi:10.1002/oby.20522

7. Peterli R, Steinert RE, Woelnerhanssen B, Peters T, Christoffel-Courtin C, Gass M et al (2012) Metabolic and Hormonal Changes After Laparoscopic Roux-en-Y Gastric Bypass and Sleeve Gastrectomy: a Randomized, Prospective Trial (Article). Obes Surg 22(5):740-748. doi:10.1007/s11695-012-0622-3

8. Lutz TA, Bueter M, Geary N (2020) Introduction to the special issue "Bariatric Surgery and Appetite". Appetite 146:104515. doi:10.1016/j.appet.2019.104515

9. Nance K, Acevedo MB, Pepino MY (2020) Changes in taste function and ingestive behavior following bariatric surgery. Appetite 146:104423. doi:10.1016/j.appet.2019.104423

10. Hankir MK, Al-Bas S, Rullmann M, Chakaroun R, Seyfried F, Pleger B (2020) Homeostatic, reward and executive brain functions after gastric bypass surgery. Appetite 146:104419.

doi:10.1016/j.appet.2019.104419

11. Azar S, Sherf-Dagan S, Nemirovski A, Webb M, Raziel A, Keidar A et al (2019) Circulating Endocannabinoids Are Reduced Following Bariatric Surgery and Associated with Improved Metabolic Homeostasis in Humans. Obes Surg 29(1):268-276. doi:10.1007/s11695-018-3517-0

12. Bryant EJ, Malik MS, Whitford-Bartle T, Waters GM (2020) The effects of bariatric surgery on psychological aspects of eating behaviour and food intake in humans. Appetite 150:104575. doi:10.1016/j.appet.2019.104575

13. Al-Najim W, Docherty NG, le Roux CW (2018) Food Intake and Eating Behavior After Bariatric Surgery. Physiol Rev 98(3):1113-1141. doi:10.1152/physrev.00021.2017

14. White JM, Piron MJ, Rangaraj VR, Hanlon EC, Cohen RN, Brady MJ (2020) Reference Gene Optimization for Circadian Gene Expression Analysis in Human Adipose Tissue. J Biol Rhythms 35(1):84-97. doi:10.1177/0748730419883043

15. Valliant MW, Tidwell DK (2007) Validation of Bioelectrical Impedance Against Dual-Energy X-Ray Absorptiometry in Adult, African American Females. J Am Diet Assoc A29. 107(8, Supplement doi:https://doi.org/10.1016/j.jada.2007.05.340

16. Frankenfield D, Roth-Yousey L, Compher $C$ (2005) Comparison of predictive equations for resting metabolic rate in healthy nonobese and obese adults: a systematic review. J Am Diet Assoc 105(5):775-789. doi:10.1016/j.jada.2005.02.005

17. Hanlon EC, Tasali E, Leproult R, Stuhr KL, Doncheck E, de Wit H et al (2016) Sleep Restriction Enhances the Daily Rhythm of Circulating Levels of Endocannabinoid 2-Arachidonoylglycerol. Sleep 39(3):653-664. doi:10.5665/sleep.5546

18. Terra X, Auguet T, Guiu-Jurado E, Berlanga A, Orellana-Gavaldà JM, Hernández M et al (2013) Longterm changes in leptin, chemerin and ghrelin levels following different bariatric surgery procedures: 
Roux-en-Y gastric bypass and sleeve gastrectomy. Obes Surg 23(11):1790-1798.

doi:10.1007/s11695-013-1033-9

19. Santiago-Fernández C, García-Serrano S, Tome M, Valdes S, Ocaña-Wilhelmi L, Rodríguez-Cañete A et al (2017) Ghrelin levels could be involved in the improvement of insulin resistance after bariatric surgery. Endocrinol Diabetes Nutr 64(7):355-362. doi:10.1016/j.endinu.2017.05.002

20. Kruljac I, Mirošević G, Kirigin LS, Nikolić M, Ljubičić N, Budimir I et al (2016) Changes in metabolic hormones after bariatric surgery and their predictive impact on weight loss. Clin Endocrinol (Oxf) 85(6):852-860. doi:10.1111/cen.13160

21. Kalinowski P, Paluszkiewicz R, Wróblewski T, Remiszewski P, Grodzicki M, Bartoszewicz Z et al (2017) Ghrelin, leptin, and glycemic control after sleeve gastrectomy versus Roux-en-Y gastric bypass-results of a randomized clinical trial. Surg Obes Relat Dis 13(2):181-188. doi:10.1016/j.soard.2016.08.025

22. Alosco ML, Spitznagel MB, Strain G, Devlin M, Cohen R, Crosby RD et al (2015) Improved serum leptin and ghrelin following bariatric surgery predict better postoperative cognitive function. $J$ Clin Neurol 11(1):48-56. doi:10.3988/jcn.2015.11.1.48

23. Jequier E (2002) Leptin signaling, adiposity, and energy balance. Ann N Y Acad Sci, 967, 379-88., http://www.ncbi.nlm.nih.gov/htbin-post/Entrez/query?db=m\&form=6\&dopt=r\&uid=12079865

24. Ostlund RE Jr, Yang JW, Klein S, Gingerich R (1996) Relation between plasma leptin concentration and body fat, gender, diet, age, and metabolic covariates. J Clin Endocrinol Metab 81(11):39093913. doi:10.1210/jcem.81.11.8923837

25. Cummings DE, Weigle DS, Frayo RS, Breen PA, Ma MK, Dellinger EP et al (2002) Plasma ghrelin levels after diet-induced weight loss or gastric bypass surgery. N Engl J Med 346(21):1623-1630. doi:10.1056/NEJMoa012908

\section{Figures}




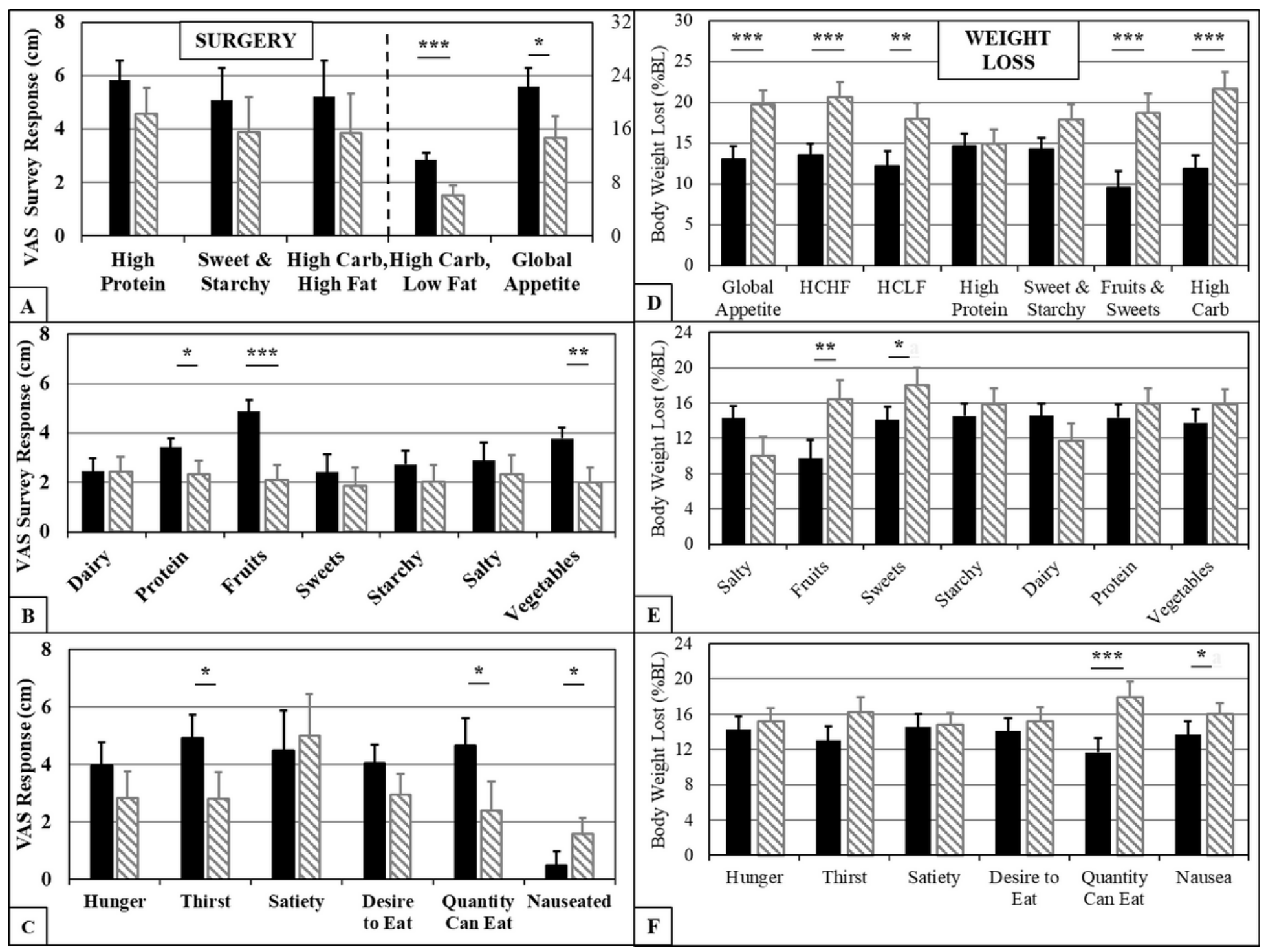

Figure 1

\section{Hunger and Appetite Ratings Pre and Post-Surgery.}

Data from eight surveys during day of identical laboratory sessions at baseline and after surgery. Survey responses were collected before and after each of four isocaloric mini-meals at baseline (black) and twelve weeks following surgery (grey stripes) for each subject $(n=10)$. Average estimated survey responses for each survey question were estimated through mixed linear modeling with subject ID as the random intercept and the effect of surgery assigned as a fixed binomial parameter. Hunger and appetite ratings at baseline and at post-surgery (A-C). (A) Composite Appetite Ratings; (B) Subjective appetite ratings for various food groups; (C) Subjective hunger ratings. Models corrected for effects of age, weight loss before or after surgery, and time of day relative to meals if present. Composite appetite ratings were summated, and then modelled separately. Significance of differences were determined by two-tailed ttests with propagated parameter uncertainties (A-C). Influence of change in hunger and appetite ratings on post-surgery weight loss (D-F). Correlations between change in subjective ratings of (D) Composite Appetite, (E) Various food groups, and (F) Hunger with weight-loss. Estimated effects were computed 
using mixed linear modeling. Models were parameterized with random factor of Subject ID, and the univariate fixed model representing the change in response between baseline (black) to post-surgery (grey stripes) to the respective question. Significance of differences were determined by two-tailed t-tests and with propagated error rates comparing expected weight loss at post-surgery associated with unchanged baseline ratings and expected weight loss at post-surgery associated with the average actual post-surgery ratings (D-F). P-values were, respectively: ${ }^{*}<0.05, * *<0.01$ and $* * * 0.001$.

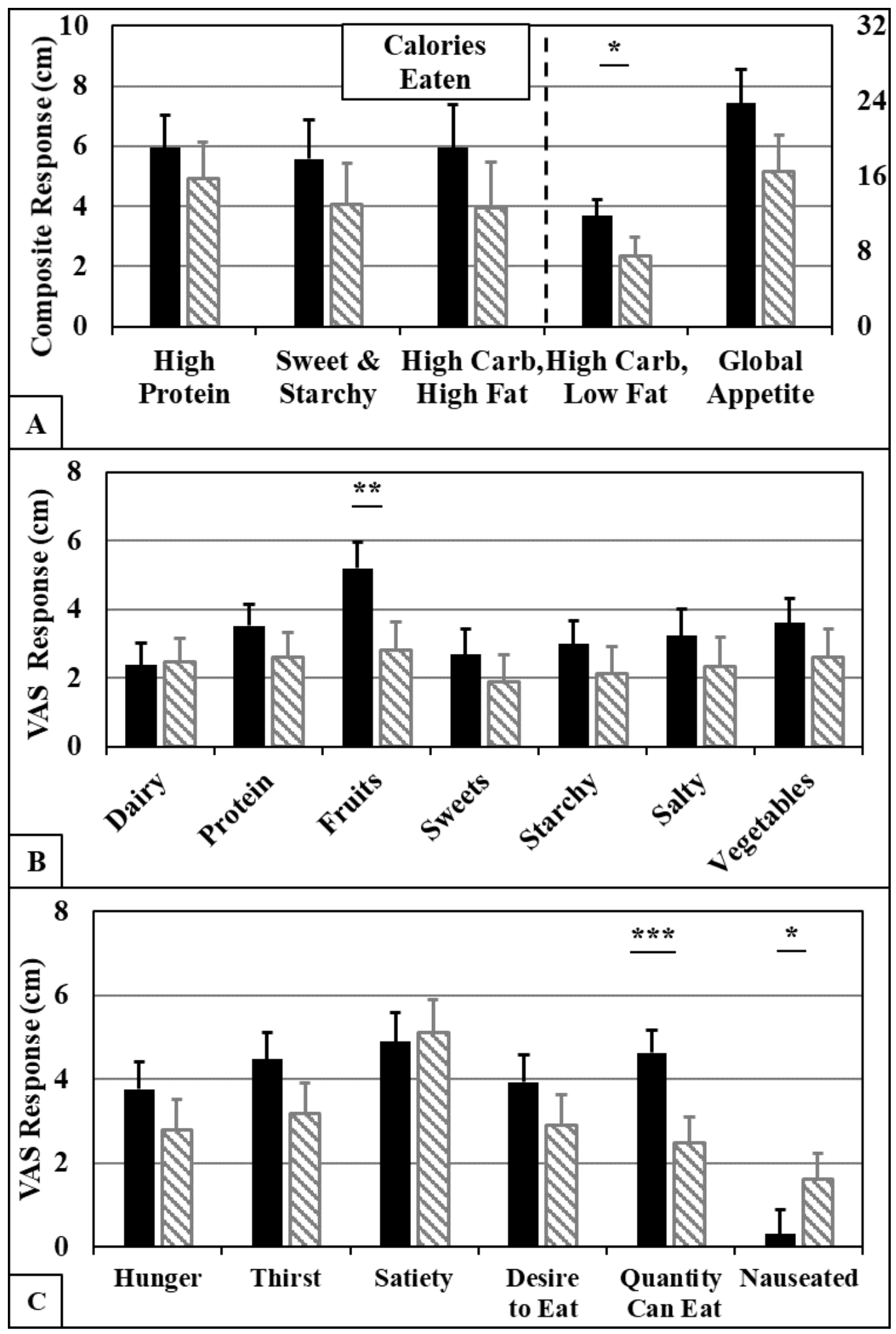


Figure 2

\section{Influence of calories eaten on hunger and appetite ratings.}

Correlations between subjective ratings and data from 8 surveys during the first day of identical laboratory sessions at baseline and after surgery. Estimated effects were computed using mixed linear modeling. Models were parameterized with random factor of Subject ID, and a univariate fixed effect based on four-meal average of calories eaten during each laboratory session. Significance determined by two-tailed t-tests with propagated error rates comparing mean VAS responses at baseline (black) to mean VAS at post-surgery (grey stripes). P-values were, respectively: $*<0.05, * \star<0.01$ and $* \star *<0.001$. 


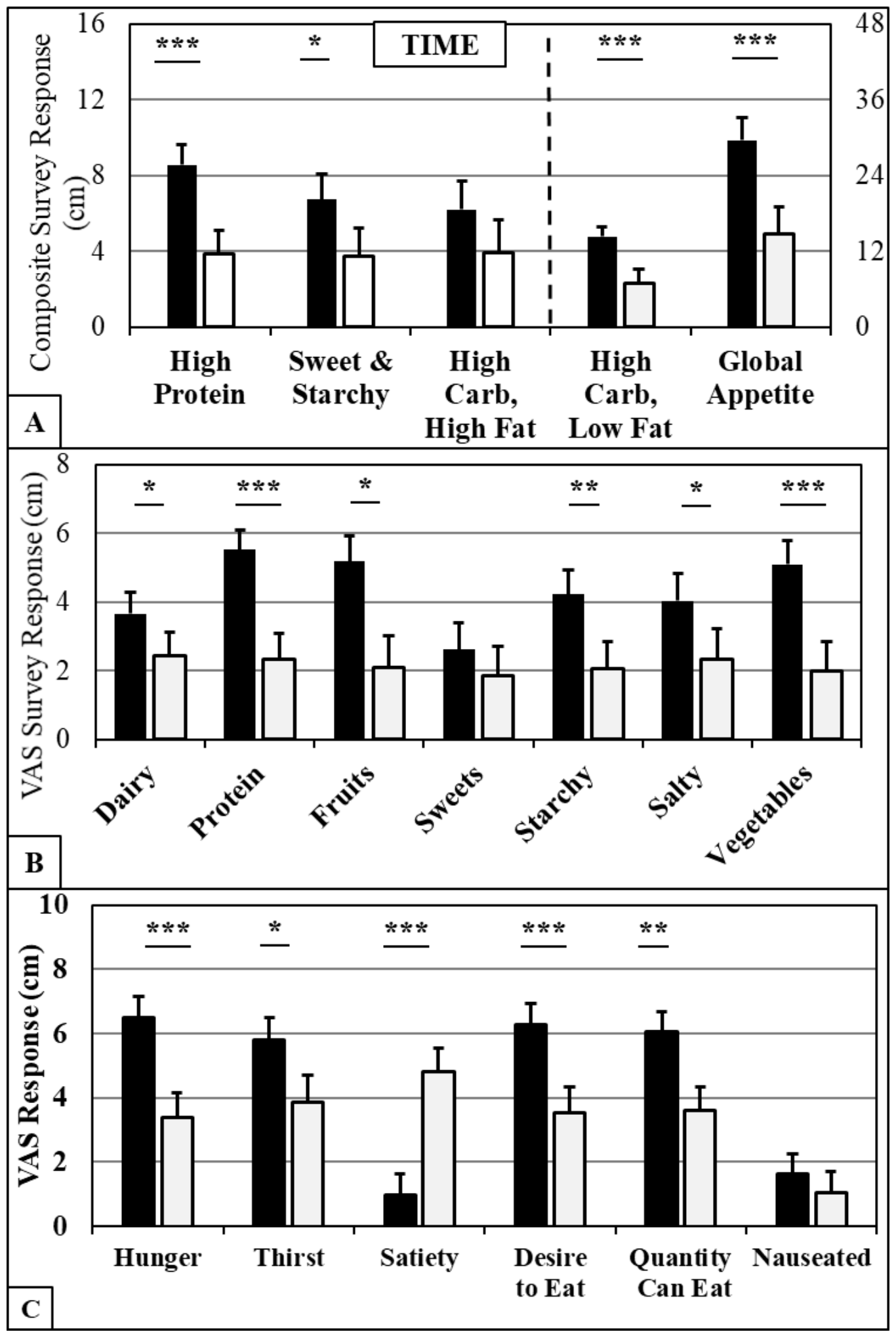

Figure 3

Influence of time on hunger and appetite ratings.

Correlations between time across day and subjective ratings of (A) Composite appetite, (B) Appetite for various food groups, and (C) Hunger. Models were parameterized with random factor of Subject ID, and bivariate fixed effects (meal, prandial). Estimated effects were computed using mixed linear modeling 
comparing responses from before the first meal (i.e. fasting; black) to responses after the last meal (i.e. end of day; white). Data was derived from the eight surveys during identical laboratory sessions at baseline and after surgery. Significance was determined by two-tailed t-tests with propagated error rates; P-values were, respectively: ${ }^{*}<0.05, * *<0.01$, and $* * *<0.001$.

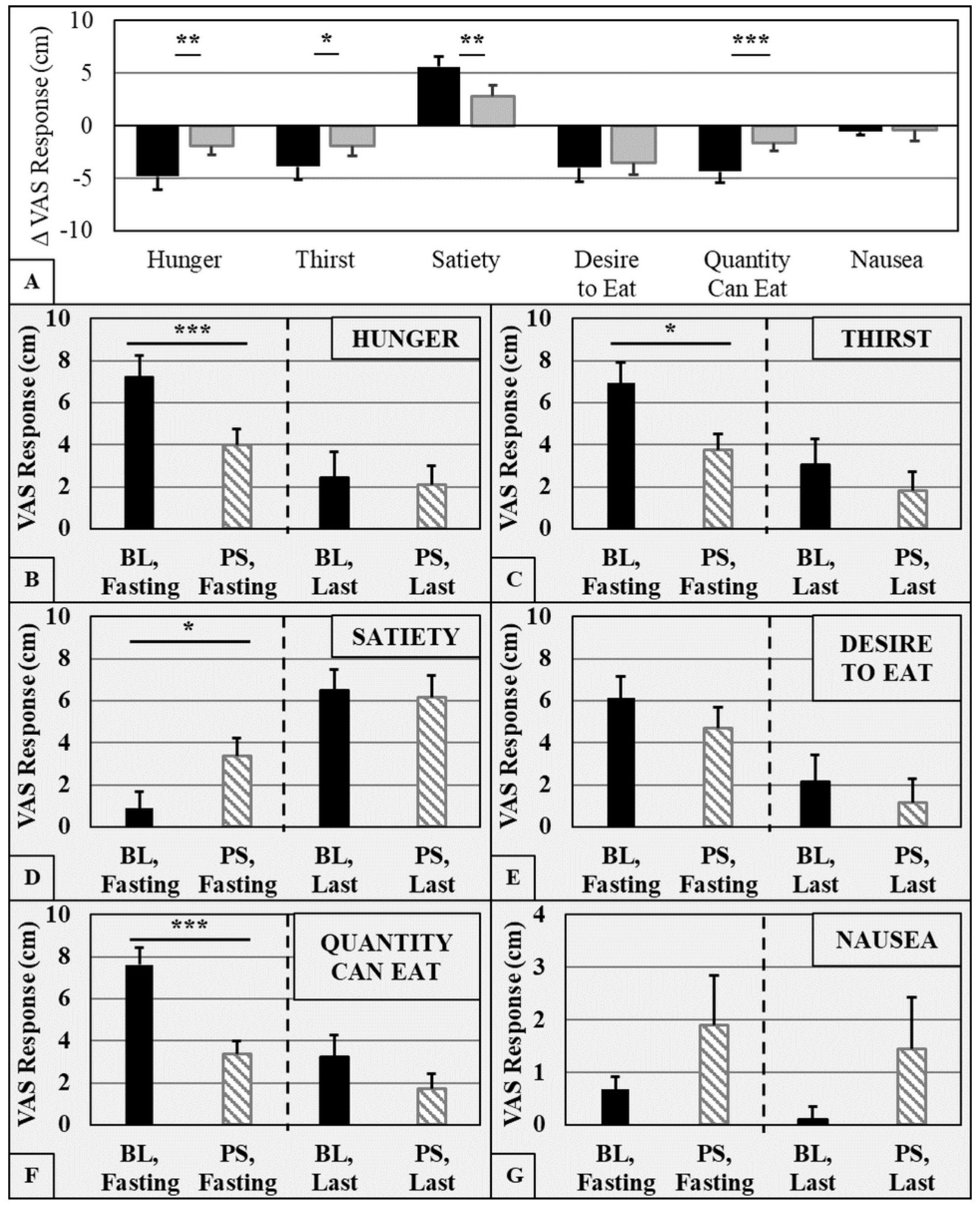

Figure 4 


\section{Analysis of hunger survey responses across time at baseline or post-surgery.}

Survey responses were collected before and after each of four isocaloric mini-meals at baseline (black, $\mathrm{BL}$ ) and twelve weeks following surgery (solid grey or grey stripes, PS). (A) The change in ratings of each estimated survey response from before the first meal to after the last meal is plotted. Significance of differences were determined by two-tailed t-test on the absolute change in responses. (B-G) Average responses were computed to estimate survey responses from before the first meal (Fasting) compared to after the last meal (Last). Mixed linear modeling was used to analyze each session data separately $(n=10)$. Significance of differences were determined by two-tailed t-test. P-values were, respectively: * $0.05, * \star<0.01$ and $\star \star \star<0.001$. 


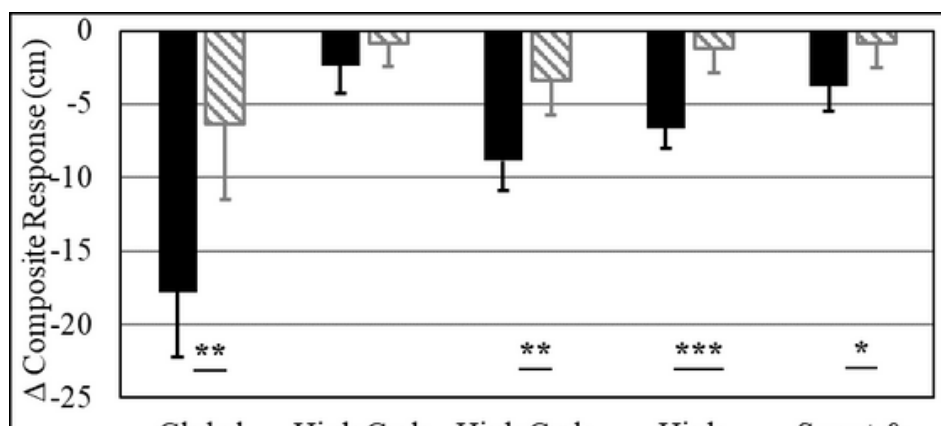

Global High Carb, High Carb, High Sweet \& A Appetite High Fat Low Fat Protein Starchy

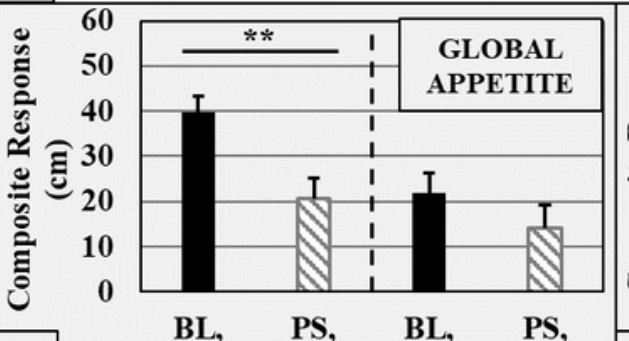

Fasting Fasting Last Last

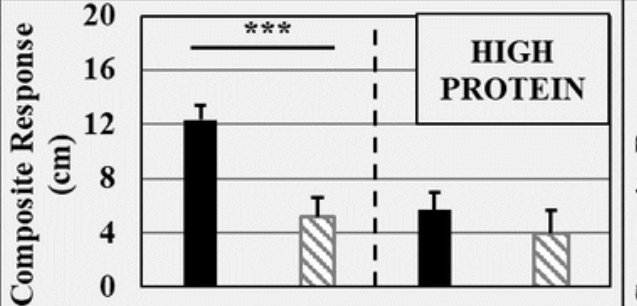

F BL, PS, BL, PS,

Fasting Fasting Last Last

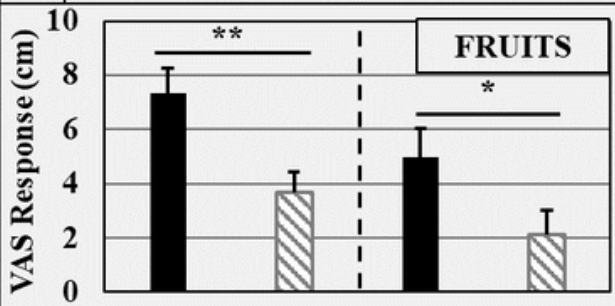

BL, PS, BL, PS,

I Fasting Fasting Last Last

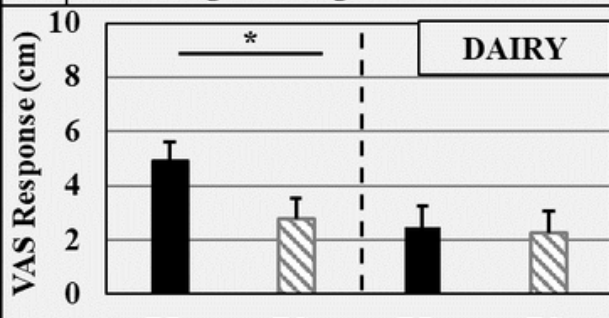

BL, PS, BL, PS,

L Fasting Fasting Last Last

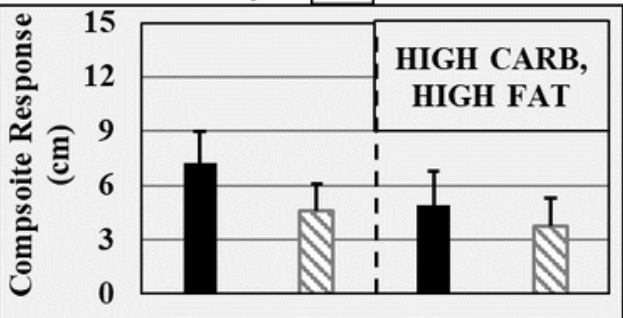

BL, PS, BL, PS,

Fasting Fasting Last Last
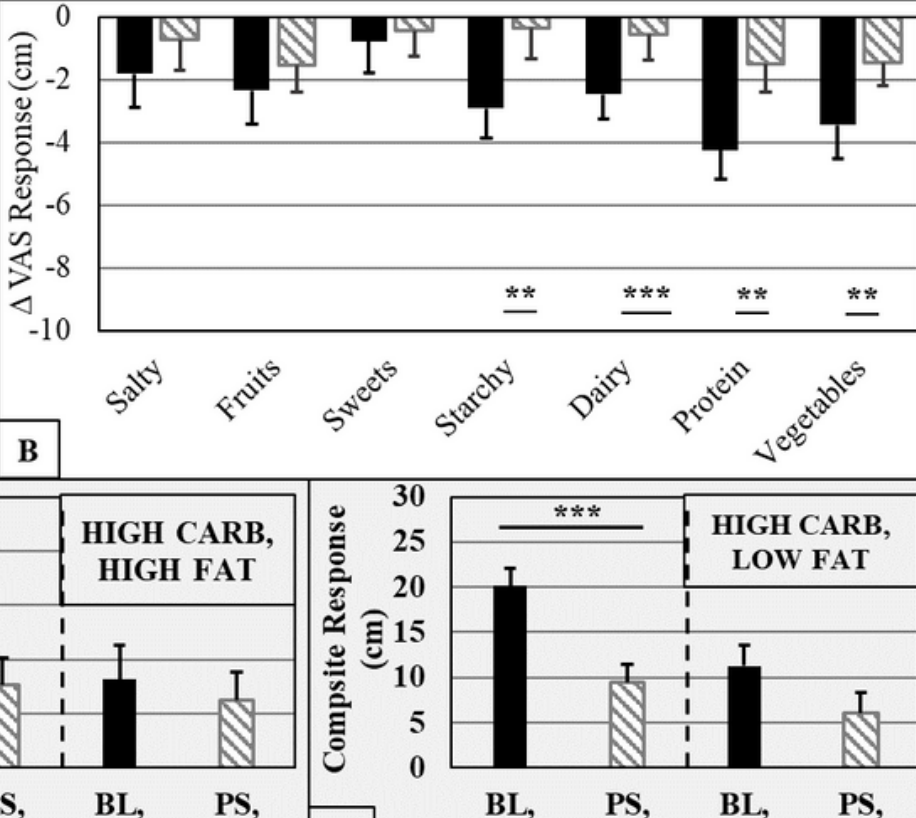

BL, PS, BL, PS,

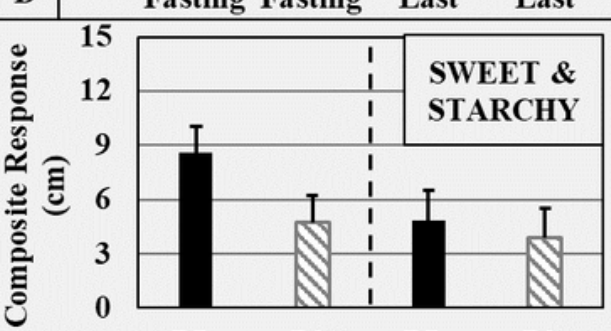

BL, PS, BL, PS,

Fasting Fasting Last Last

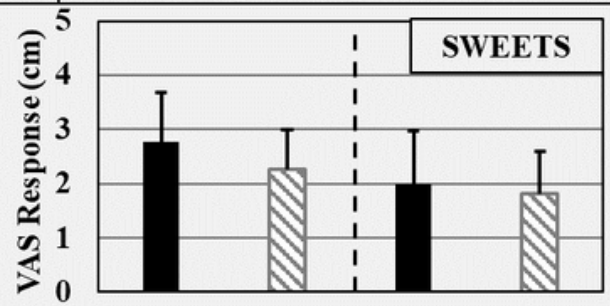

BL, PS, BL, PS,

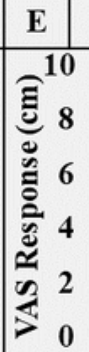

Fasting Fasting

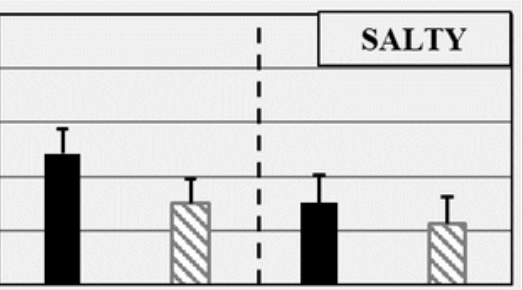

BL, PS, BL, PS,

Fasting Fasting Last Last

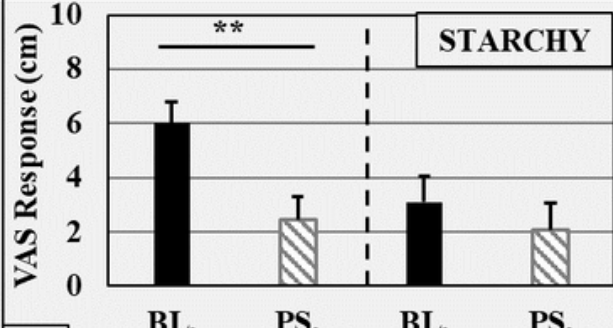

Fasting Fasting Last Last
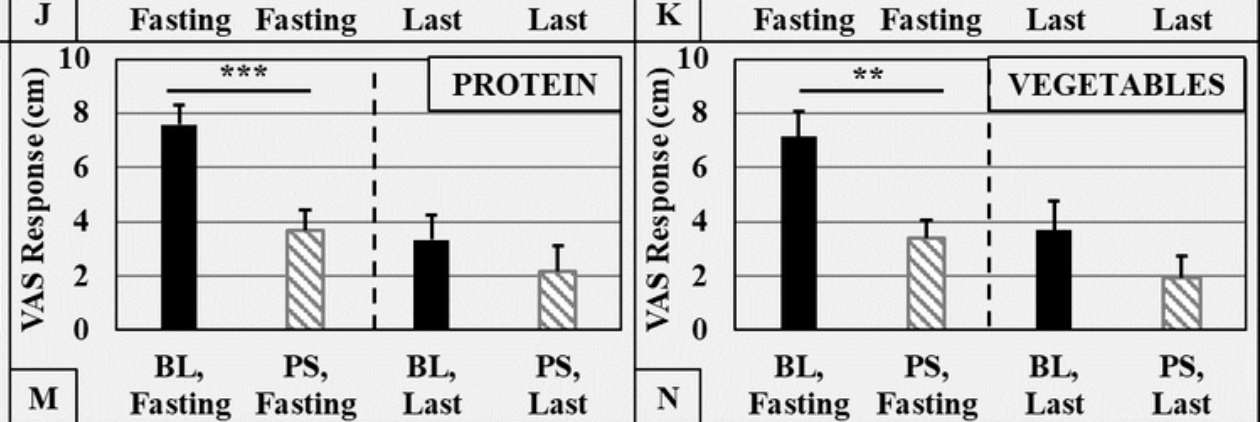

Figure 5

Analysis of appetite survey responses across time at baseline or post-surgery.

Survey responses were collected before and after each of four isocaloric mini-meals at baseline (black, PS) and twelve weeks following surgery (grey stripes, PS). (A, B) The change in each estimated survey response from before the first meal until after the last meal is plotted. Significance of differences were 
determined by two-sided t-test on the absolute change in either simple or composite responses. (C-N) Average responses were computed to estimate fasting responses, before the first meal, and after the last meal. Mixed linear modeling was used to analyze each session data separately $(n=10)$. Significance of differences were determined by two-tailed t-test. P-values were, respectively: ${ }^{*}<0.05, * \star<0.01$ and $* \star *<$ 0.001 .

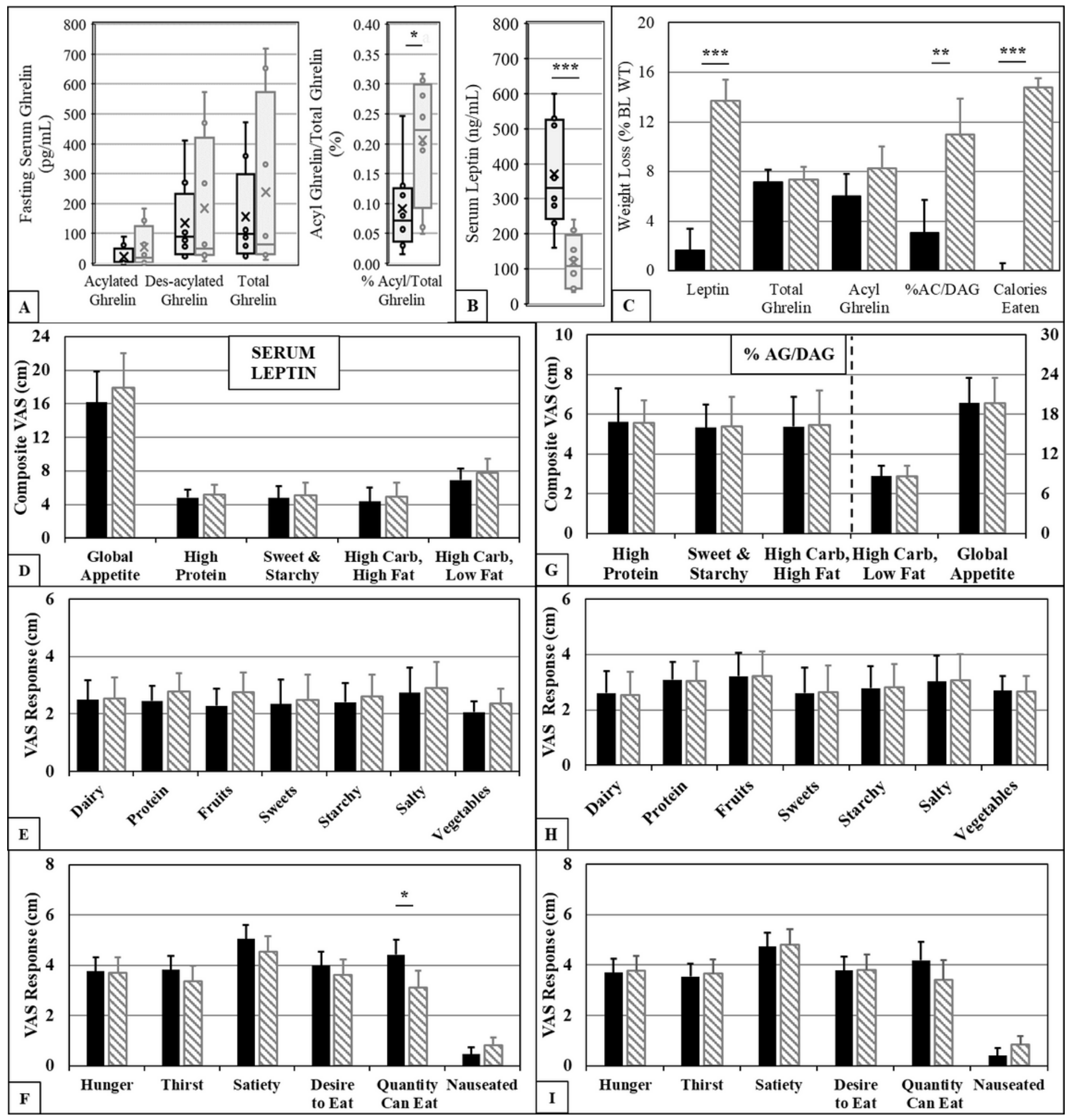

Figure 6 


\section{Serum Levels of ghrelin and leptin and influence on hunger and appetite ratings.}

Fasting serum was collected $(\mathrm{N}=8)$ during the morning prior to the first meal at baseline and at twelve weeks post-surgery. Hormones measured were: acylated ghrelin and total ghrelin via ELISA (Millipore Sigma EZGRT-89K, EZGRA-88K), and leptin via radioimmunoassay (Millipore Sigma HL-81K). (A-B) Fasting serum hormone levels at baseline (black), or post-surgery (grey or grey stripes). Significance of differences was determined by two-tailed paired t-test with propagated error rates comparing hormone levels at baseline and at post-surgery. (C) Linear modeling to estimate percent weight lost using respective single fixed factor of respective hormone levels, the ratio of acyl-ghrelin to total ghrelin, or calories eaten across four meals of laboratory session at baseline (black) or post-surgery (grey stripes). Significance of differences was determined by two-tailed paired t-test with propagated error rates comparing estimated weight loss at post-surgery associated with unchanged baseline levels or with the average of actual post-surgery levels of the respective measures (A-C). The influence of serum leptin and active ghrelin activity on hunger and appetite ratings in shown in D-I. Data from eight surveys during the first day of identical laboratory sessions at baseline and after surgery. Estimated effects were computed using mixed linear modeling. Models were parameterized with random factor of Subject ID, and a univariate fixed effect based on: (D-F) fasting serum leptin and (G-I) percent ratio of acyl-ghrelin to total ghrelin in fasting serum. Significance determined by two-tailed t-tests with propagated error rates comparing mean VAS responses at baseline (black) to mean VAS responses at post-surgery (grey stripes). P-values were: $*<0.05, * *,<0.01, * \star *<0.001$. 